\title{
Comparison of Excitation Signals in Active Magnetic Bearing System Identification
}

\author{
Jouni Vuojolainen ${ }^{1}$ Niko Nevaranta ${ }^{1}$ Rafal Jastrzebski ${ }^{1}$ Olli Pyrhönen ${ }^{1}$ \\ ${ }^{1}$ Department of Electrical Engineering, Lappeenranta University of Technology, FI-53851 Lappeenranta, Finland \\ E-mail: jouni.vuojolainen@lut.fi, niko.nevaranta@lut.fi, rafal.jastrzebski@lut.fi, olli.pyrhonen@lut.fi
}

\begin{abstract}
Active magnetic bearings (AMBs) offer frictionless suspension, vibration insulation, programmable stiffness, and damping, among other advantages, in levitated rotor applications. However, AMBs are inherently unstable and require accurate system models for the high-performance model-based multi-input multi-output control of rotor position. Control electronics with high calculation capacity and accurate sensors of AMBs provide an opportunity to implement various identification schemes. A variety of artificial excitation signal-based identification methods can thus be achieved with no additional hardware. In this paper, a selection of excitation signals, namely the pseudorandom binary sequence (PRBS), chirp signal, multisine, and stepped sine are presented, applied, and compared with the AMB system identification. From the identification experiments, the rotor-bearing system, the inner current control loop, and values of position and current stiffness are identified. Unlike recently published works considering excitation-based identification of AMB rotor systems, it is demonstrated that identification of the rotor system dynamics can be carried out using various well-established excitation signals. Application and feasibility of these excitation signals in AMB rotor systems are analyzed based on experimental results.
\end{abstract}

Keywords: Active magnetic bearings (AMB), magnetic levitation, chirp signal, frequency-domain analysis, multisine, pseudorandom binary sequence (PRBS), stepped sine, system identification

\section{Introduction}

Excitation signal-based identification routines are of key importance in the commissioning phase of active magnetic bearing (AMB) levitated rotor systems. Accurate models obtained by system identification are needed in order to design high-performance controllers (Noshadi et al., 2016), and they have an increasingly important role for diagnostic (Schuhmann et al., 2012) and monitoring purposes (Quinn et al., 2005), (Aenis et al., 2002), (Tiwiri and Chougale, 2014). A common choice for the excitation signal is a sine-wave-based spectrally rich signal, such as a multisine or swept sinusoid signal with a frequency content covering the desired frequency bands. In addition, a stepped sine is a typical signal choice for AMB commissioning purposes to guarantee rich excitation at a specific frequency at the time.

In the literature covering either modeling or control issues of AMB-levitated rotor systems, sine-wave-based excitation signals have been widely applied in identification experiments to obtain an adequate model for the system dynamics. In (Smirnov, 2012), an automatic commissioning approach for an AMB system has been proposed where stepped sine has been considered as an excitation signal to identify frequency responses. Similarly, in (Ahn et al., 2003), stepped sine has been applied for closed-loop identification to obtain a rigid body model for controller design. In (Hynynen and Jastrzebski, 2009), (Hynynen et al., 2010), optimized multisine signals have been proposed for closed-loop rotor system identification to avoid har- 
monics produced by a nonlinear system. Although the commissioning and monitoring of an AMB-supported motor system is mostly based on the use of stepped sine or multisine (Smirnov, 2012)-(Kulesza, 2014), when moving towards advanced online identification routines, other signals such as the pseudorandom binary sequence (PRBS) and chirp should also be considered. Despite the extensive research in the field of closed-loop identification of AMB systems, there are only a few studies available where other excitation signals have been applied, or their feasibility to AMB system identification has been discussed. In (Vuojolainen et al., 2016), (Jastrzebski et al., 2016b), the PRBS has been introduced to study high-frequency bending modes in rotor dynamics. In addition, in (Garcia et al., 2016), PRBS is used for performance assessment of a bearingless motor to identify sensitivity functions. Moreover, in (Noshadi et al., 2016), (Inman et al., 2005), (Wroblewski et al., 2012), (Fang et al., 2013), (Tang et al., 2016), and (Lanzon and Tsiotras, 2005), chirp has been applied for the identification of rotor system dynamics. However, these studies do not provide any analysis of the obtained frequency responses as the main focus is on the identification for control, in other words, the models are evaluated to be representative in frequency regions that are relevant for control design purposes.

Despite the wide practical application of excitationbased identification routines for AMB systems, it appears that no papers have compared or discussed the applicability of different excitation signals for such a complex plant. In this paper, the feasibility of different types of excitation signals for identification of an AMBlevitated rotor system is studied. The excitation signals under consideration are PRBS, chirp signal, multisine, and stepped sine. An experimental six-degree-offreedom modular AMB rotor system is taken as a test case machine. Based on the experimental identification results obtained with various excitation signals, the rotor dynamics, the inner current control loop dynamics, and the position stiffness and current stiffness values are identified for time-continuous linear models. These values are used to validate the identification results, and more importantly, a comparison between the different excitation signals is provided for the first time for such a comprehensive and complex plant model. Moreover, the identified position and current stiffness values are compared with static measurements using a force gauge, which is another key contribution of this paper. It is pointed out that the results presented in this paper are of importance in coupled AMB rotor model identification, levitation system diagnostics, and monitoring.

The paper is constructed as follows. First, the prop- erties of all the excitation signals under study, namely the PRBS, chirp signal, multisine, and stepped sine are introduced and described in Section 2. After that, an identification problem of an active magnetic bearing system is discussed in Section 3 in brief. In Section 4, the experimental conditions for all excitation signals are given and identification results for the rotor-bearing system, inner current control loop, and position and current stiffness values are reported. Finally, conclusions are drawn by comparing the frequency responses and the estimated stiffness values obtained from the identification experiments with different excitation signals.

\section{Excitation signals}

Sufficiently rich excitation signals, in other words, generators of persistent excitation are of key importance to guarantee informative input-output data for system identification. Naturally, depending on the identification problem, distinct criteria direct the choice of the excitation signal Pintelon and Schoukens (2012). This paper studies closed-loop identification of an AMBlevitated system using various excitations signals.

\subsection{PRBS}

Pseudorandom binary sequence (PRBS) shown in Fig. 1 a) is an excitation signal that is deterministic and periodic and varies between two levels, amplitudes $+A$ and $-A$. The PRBS is easy to generate, and it has controllable spectral energy, a high spectral energy over a wide band range, and an optimal spectrum for the excitation signal. The basic PRBS is defined by the number of cells $d$ and the selected excitation frequency $f$. With the number of cells $d$, the length $N$ of the PRBS is calculated as

$$
N=2^{d}-1 .
$$

The frequency resolution $f_{r}$ of the PRBS is calculated by

$$
f_{r}=\frac{f_{s}}{N} .
$$

where $f_{s}$ is the sampling frequency. The number of data points $L$ needed to save the PRBS identification data is

$$
L=\frac{N f_{s}}{f} .
$$

The maximum length $N$ is limited by the maximum available $L$ and the ratio $f_{s} / f$, as seen in (3). Now, if we want to increase $f_{r}$ defined by (2), this can be achieved with a higher $f_{s}$, a higher $f$, and a lower $N$. 


\subsection{Chirp}

Chirp signal (see Fig. 1 b) ), also known as sweep or swept signal, is an identification excitation signal in which the frequency is swept up or down in one period. Chirp signals are typically divided into two groups: linear, in which the frequency of the signal varies linearly with time, and exponential/geometrical, in which the frequency varies with geometric progression. In this paper, a linear chirp signal with a sinusoidal waveform is applied. The instantaneous frequency of the linear chirp signal can be calculated by

$$
f(t)=f_{0}+k t
$$

where $f_{0}$ is the starting frequency, $t$ is the current time, and $k$ is the rate of frequency change, which can be obtained by

$$
k=\frac{f_{1}-f_{0}}{T},
$$

where $f_{1}$ is the final frequency and $T$ is the final time after the sweep from $f_{0}$ to $f_{1}$. The equation of the sine chirp signal is Pintelon and Schoukens (2012)

$$
u(t)=A \cdot \sin \left(2 \pi \cdot\left(f_{0} t+\frac{k}{2} t^{2}\right)\right),
$$

where $A$ is the amplitude of the chirp signal. Chirp signals have been widely applied in AMB identification owing to their main advantage Inman et al. (2005)Lanzon and Tsiotras (2005): at least to some degree, the frequency content of the signal can be controlled, and hence, its power can be concentrated on a specific frequency range, in other words, regions that are relevant for control design purposes.

\subsection{Multisine}

A multisine signal depicted in Fig. 1 c), with phases of the sine waves chosen randomly from the interval $[0$, $2 \pi$ ], can be determined by

$$
u(t)=\sum_{n=1}^{N_{\mathrm{f}}} A_{n} \cdot \cos \left(2 \pi f_{n} t+\phi_{n}\right),
$$

where $N_{f}$ is the total number of frequencies over $n$ indices, $A_{n}$ is the amplitude, $f_{n}$ is the frequency, and $\phi_{n}$ is the phase of the $n$th sine wave component. When applied to AMB system identification, the multisine signal must be carefully designed as discussed in Hynynen and Jastrzebski (2009), Hynynen et al. (2010), where the excitation signal amplitude content has been designed to avoid the harmonics produced by the nonlinearities in the system. By adopting the same guidelines, the multisine signal implemented in this study is generated by using a maximum of four bands, where the starting and final frequencies, the frequency resolution, and the amplitude can be chosen freely for each band.

\subsection{Stepped Sine}

Stepped sine is a type of sine wave that has all the power at one frequency only as illustrated in Fig. 1 d). Therefore, several frequencies have to be excited separately to cover the required frequency range. Each component has a frequency $f_{n}$ and an amplitude $A_{n}$, which, in general, can be chosen freely. The equation of the stepped sine wave is

$$
u(t)=A_{n} \cdot \sin \left(2 \pi \cdot f_{n} t\right)
$$

In this paper, an adaptive amplitude stepped sine is considered where the frequency $f_{n}$ is selected freely, but the amplitude $A_{n}$ is adjusted to get an acceptable response and to keep the system in the linear region Smirnov (2012). The method is based on prespecified minimum and maximum limits in which the amplitude of the signal is controlled until a desirable value is reached.

\section{Identification of an Active Magnetic Bearing System}

Owing to its unstable open-loop system dynamics, an active-magnetic-bearing-supported rotor system is a typical example of a closed-loop identification problem. Naturally, the excitation signal can be superposed to different locations of the control structure, that is, the reference or controller output, in order to identify an open-loop plant model or analyze different properties (sensitivities) of the achieved closed-loop system Larsonneur (2009).

In Fig. 2, a block diagram of the closed-loop controlled AMB system illustrates the excitation and signal locations considered in this paper, where $r_{u}$ denotes the excitation signal at the position control output, which is summed with the control current constituting the plant input $u_{1}$. The measured current of the AMB is denoted by $y_{1}$ and the rotor position by $y_{2}$.

It is emphasized that the AMB system identification must be carried out during system levitation, when the feedback control is operating actively Larsonneur (2009). In this paper, the transfer functions are identified by a direct approach, where the influence of the feedback is omitted. The main advantage of this approach is that the knowledge of the controller is not needed, but depending on the identification problem, it has the disadvantage leading to biased estimates 
a) PRBS
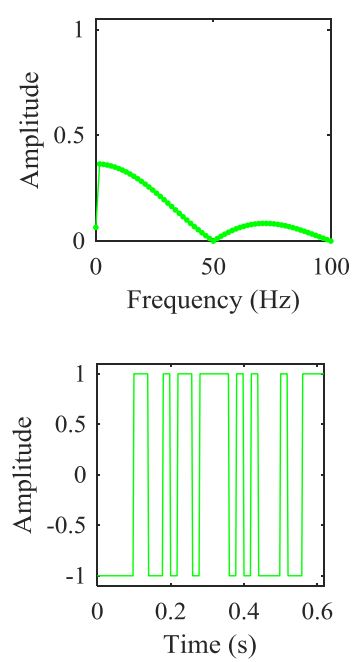

b) Chirp
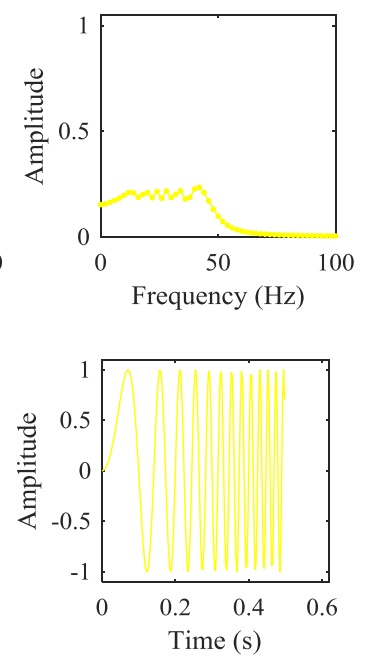

c) Multisine
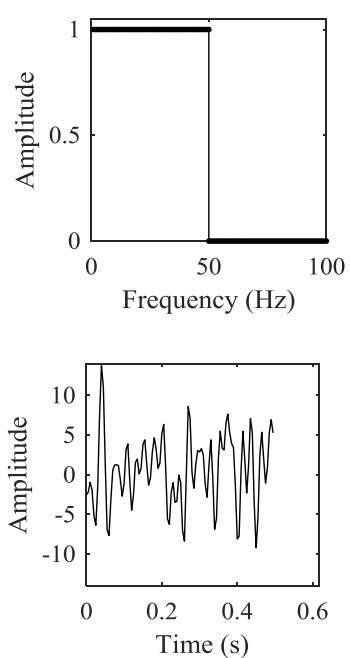

d) Stepped sine
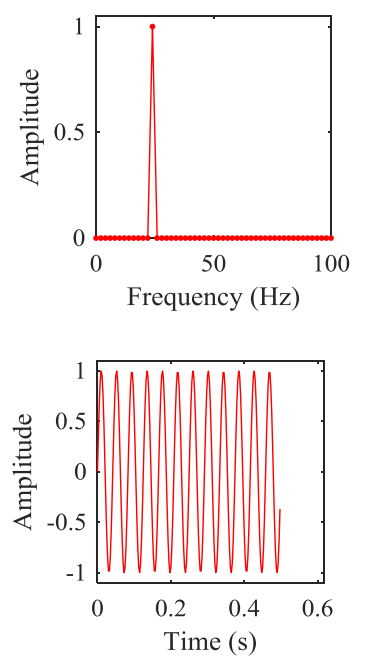

Figure 1: Comparison of the excitation signals in the frequency (upper row) and time domains (bottom row).

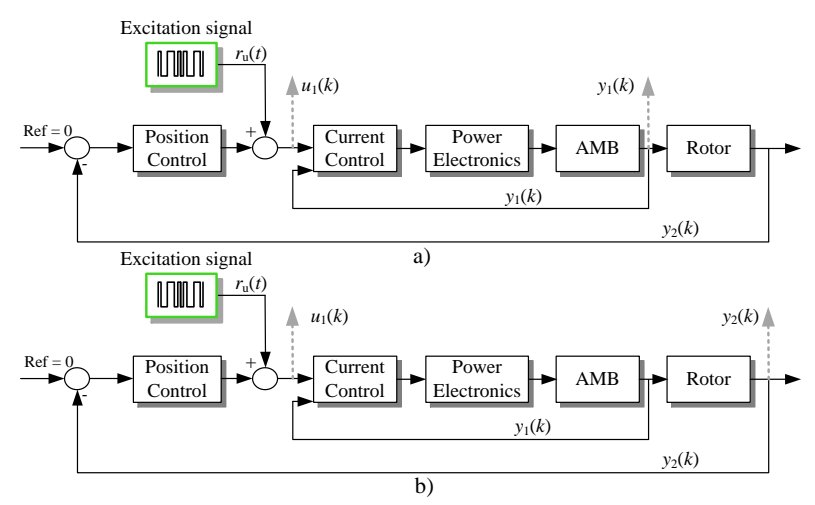

Figure 2: Block diagram of the AMB system with input and output signals used in the identification (dotted grey lines). a) Identification of bearing (current control loop) dynamics using the current reference signal $u_{1}(k)$ and the measured current $y_{1}(k)$ and b) identification of rotor-bearing dynamics using the current reference signal $u_{1}(k)$ and the measured position signal $y_{2}(k)$.

in some cases. Alternatives for a direct approach include an indirect approach and a joint-input-output approach Hynynen (2011). In the indirect approach, the closed-loop transfer function from the reference signal to the plant output is estimated. In the joint-inputoutput approach, the transfer function is estimated with the reference signal, and both the plant input and output are considered as outputs. It is pointed out that direct identification has provided accurate models for controller design of an AMB system in several papers Aenis et al. (2002), Smirnov (2012), Vuojolainen et al. (2016), Jastrzebski et al. (2016b), and Wroblewski et al. (2012), and therefore, the same approach is considered here. Moreover, the empirical transfer function estimation (ETFE) is used for the transfer function identification in this paper. The transfer functions are estimated for the SISO case only, that is, by exciting one current reference input of the system and then the dynamics are identified from the measured outputs.

\section{Experimental Results}

An AMB test rig is used to test PRBS, chirp signal, multisine, and stepped sine excitation signals. The AMB test rig consists of two radial and one axial AMB, resulting in a six-DOF system. The picture of the test rig is shown in Fig. 3. The test rig is discussed in detail in Jastrzebski et al. (2016b), where the stable rotational operation is shown.

All excitation signals are generated by Simulink in the Beckhoff TwinCAT environment. The Beckhoff TwinCAT environment is described in more detail in Jastrzebski et al. (2016a). The PRBS, chirp signal, and multisine are limited to a one-second period based on the memory consumption considerations, and the number of periods of the excitation sequence has to be at least two. This is because the first period is the transient period, and the data points related to this 


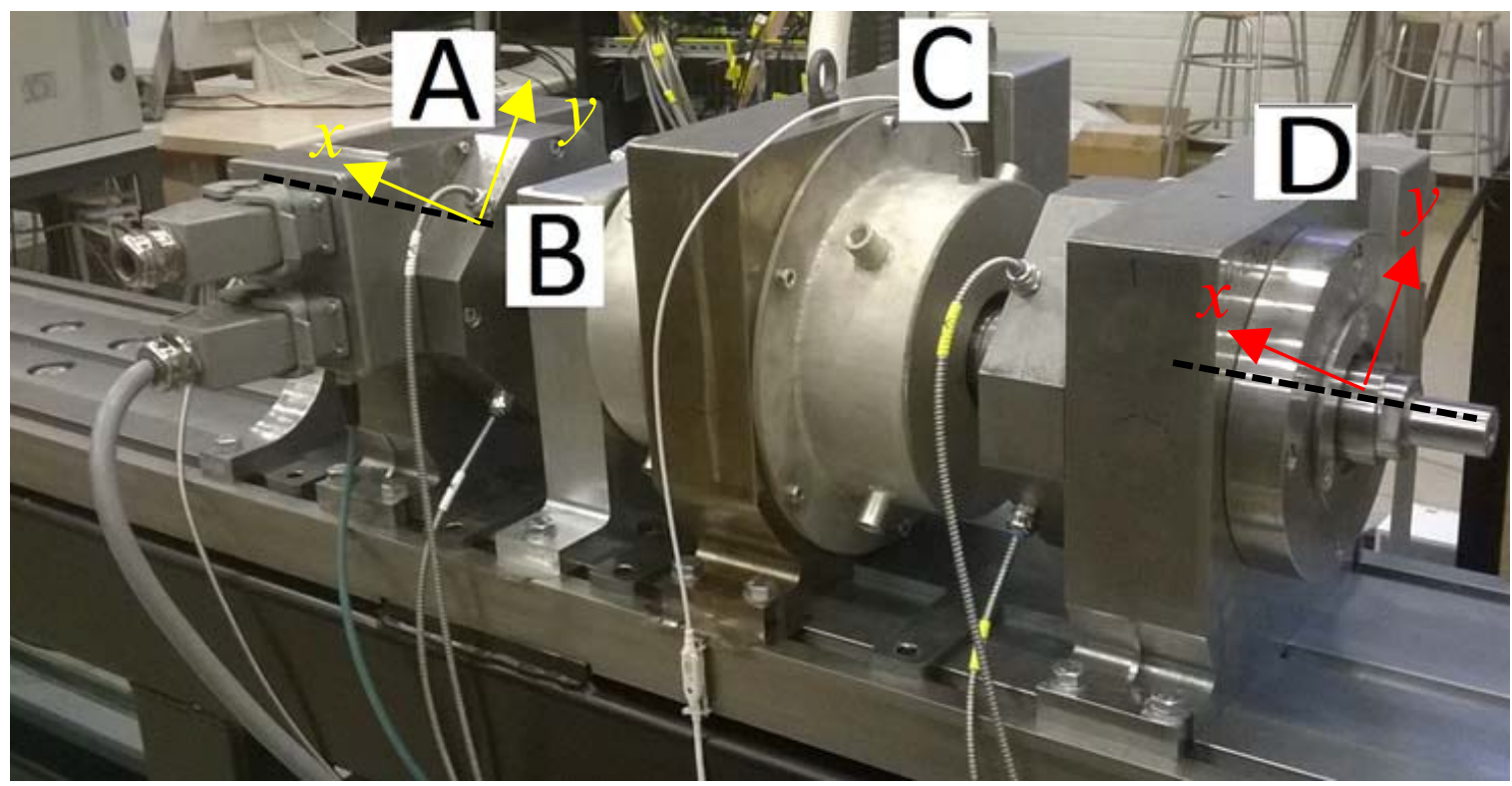

Figure 3: Experimental test rig. A) radial bearing non-drive-end, B) axial bearing, C) induction motor, and D) radial bearing drive-end.

period are discarded as they lead to incorrect results. Naturally, if three or more periods are used, an average over the periods is calculated and saved to increase the signal to noise ratio (SNR) and to minimize the effect of measurement errors.

In this paper, the excitation signals are designed with the following properties. The PRBS signal has a frequency of $3.33 \mathrm{kHz}$ with eleven cells, resulting in a frequency resolution of $1.63 \mathrm{~Hz}$. The amplitude is 2.5 $\mathrm{A}$, and ten periods are collected. The chirp signal has a starting frequency of $5 \mathrm{~Hz}$ and a final frequency of $1 \mathrm{kHz}$. The amplitude is chosen as $1.0 \mathrm{~A}$, and a onesecond sweep and ten periods are considered. Again, the multisine signal has four bands, and ten periods are recorded. The first band is from $1 \mathrm{~Hz}$ to $19 \mathrm{~Hz}$ in $2 \mathrm{~Hz}$ steps with an amplitude of $10 \mathrm{~mA}$. The second band is from $23 \mathrm{~Hz}$ to $199 \mathrm{~Hz}$ in $4 \mathrm{~Hz}$ steps, the amplitude being $50 \mathrm{~mA}$. The third band is from $203 \mathrm{~Hz}$ to $399 \mathrm{~Hz}$ in $2 \mathrm{~Hz}$ steps, and the amplitude is $80 \mathrm{~mA}$. Finally, the fourth band is from $403 \mathrm{~Hz}$ to $698 \mathrm{~Hz}$ in $5 \mathrm{~Hz}$ steps, and the amplitude is set to $120 \mathrm{~mA}$. The stepped sine has a frequency range from $1 \mathrm{~Hz}$ to $750 \mathrm{~Hz}$, and 250 points are used. Thus, the frequency resolution is 3.01 $\mathrm{Hz}$ while the maximum amplitude is $2.5 \mathrm{~A}$.

\subsection{Rotor-Bearing System Identification}

To identify the rotor-bearing system, excitation is applied at the reference control current as depicted in Fig. 2 b). The plant input $u_{1}(k)$ and output $y_{2}(k)$ signals are measured for the corresponding axis, and the open-loop transfer function from the reference control current to the rotor displacement is obtained. This open-loop transfer function can be written as

$$
G(\mathrm{j} \omega)=\frac{Y_{2}(\mathrm{j} \omega)}{U_{1}(\mathrm{j} \omega)},
$$

where $Y_{2}(j \omega)$ and $U_{1}(j \omega)$ represent the Discrete Fourier Transform (DFT) of the input and output signals. After the open-loop plant transfer functions have been obtained according to all of the identification results with different excitation signals, a comparison is made with the rotor transfer function of the Finite Element Method (FEM) model. In this paper, the FEM model is considered as a mathematical reference model that is based on the rotor dimensions. The obtained frequency responses from the identification experiments using different excitations are shown in Fig. 4. In addition, a zoom is taken from the frequency range $\{220$, $800\} \mathrm{Hz}$ covering the flexible dynamics to compare the estimated frequency response.

Based on the identified frequency responses on the drive-end $\mathrm{x}$ - and $\mathrm{y}$-axes (DX and DY), all excitation signals yield a similar accuracy compared with the FEM model on the slope to the first resonance frequency (frequency range from $10 \mathrm{~Hz}$ to $280 \mathrm{~Hz}$ ). As expected, after the first resonance frequency, all the estimated frequency responses start to diverge from the FEM model results. Nevertheless, it is important to notice that the identification tests carried out with different excitation signals are still able to identify the 
a) DX

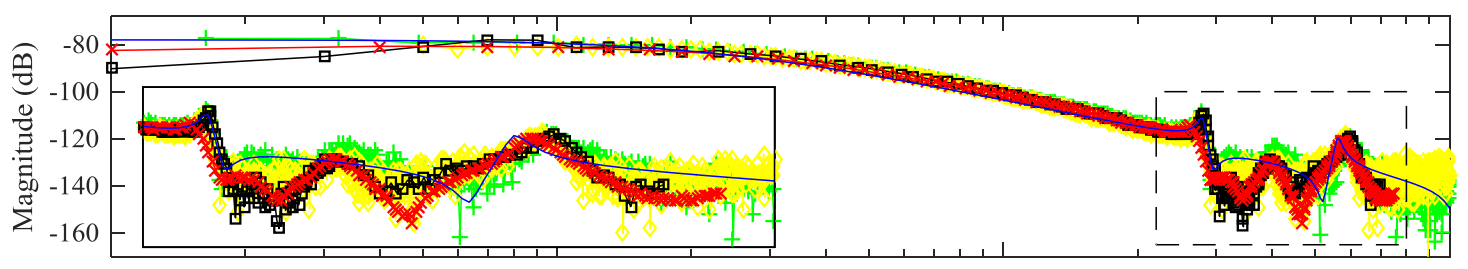

b) DY

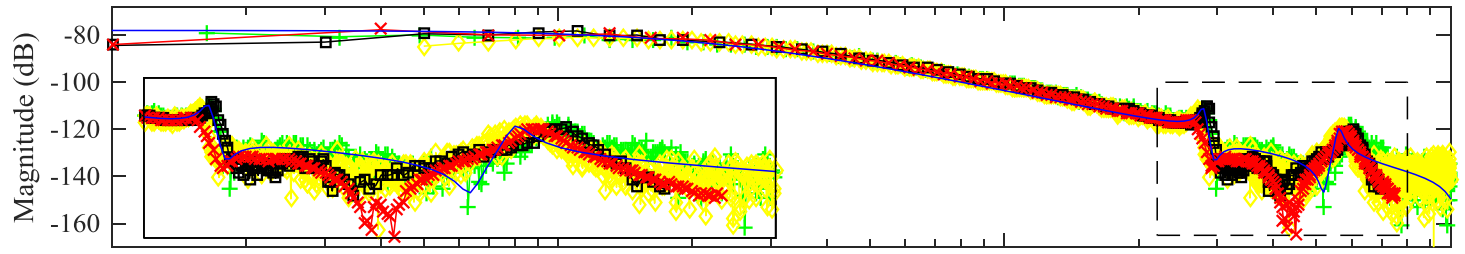

c) NX

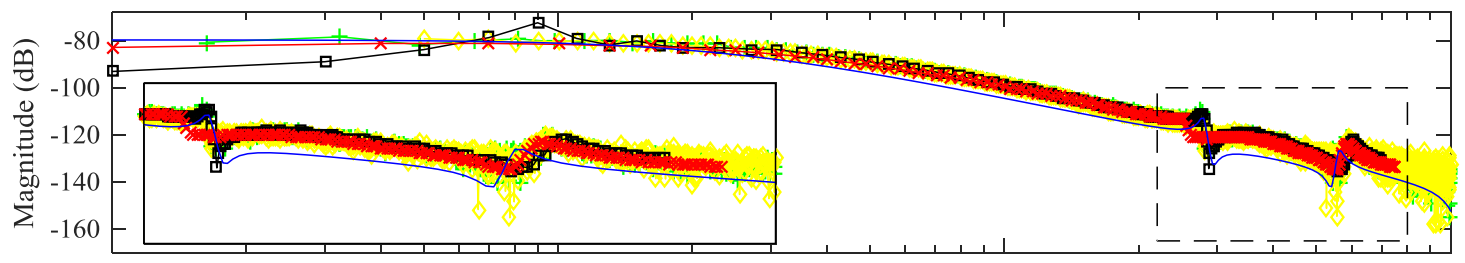

d) NY

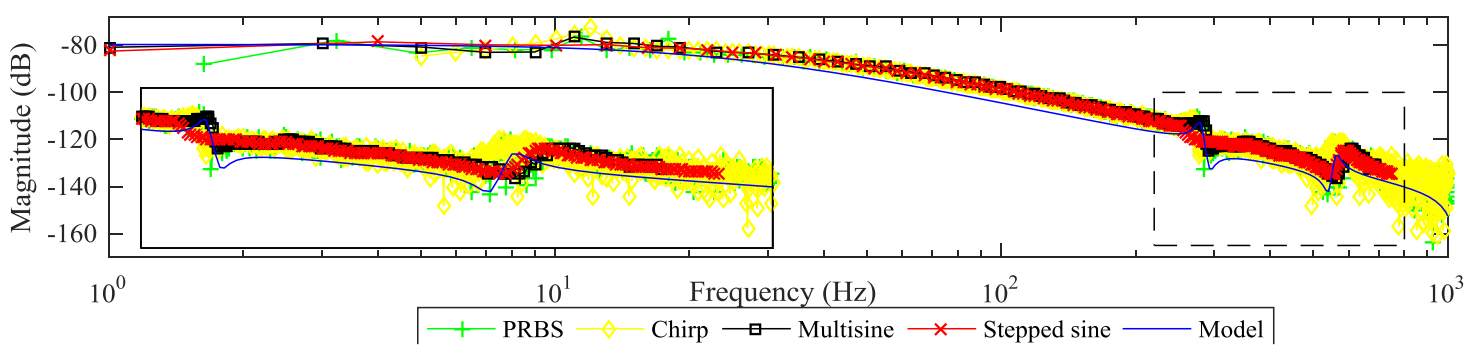

Figure 4: Comparison of the open-loop transfer functions of the rotor-bearing system. DX and DY denote the drive-end $\mathrm{x}$-axis and $\mathrm{y}$-axis, and NX and NY denote the non-drive-end $\mathrm{x}$-axis and $\mathrm{y}$-axis, respectively.

second resonance frequency located at $560 \mathrm{~Hz}$. In the frequency range from $1 \mathrm{~Hz}$ to $10 \mathrm{~Hz}$, which refers to the DC gain values, there is some variation between the identification methods.

Again, based on the identification results given in Fig. 4 c) and d), all of the tested excitation signals yield a similar accuracy from the slope to the second resonance frequency compared with the FEM model on the non-drive-end $\mathrm{x}$ - and $\mathrm{y}$-axes (NX and NY). However, there is a noticeable offset between the FEM model and the identification results in this frequency range. Reasons for this are the nonlinear gain and steady-state errors in the inner current control loops and the uncertain transportation and PWM delay in the applied industrial drives connected through the CAN bus. Variation in the parameters of the inner current control loops is observed in the explicit inner-loop frequency-based identification and the static identification.

\subsection{Inner Current Control Loop Identification}

In the inner current control loop identification, excitation is applied at the reference control current as depicted in Fig. 2 a). The plant input $u_{1}$ and output $y_{1}$ signals are measured for the corresponding axis, and the open-loop transfer function from the reference control current to the rotor displacement is generated. This transfer function can be written as

$$
G(\mathrm{j} \omega)=\frac{Y_{1}(\mathrm{j} \omega)}{U_{1}(\mathrm{j} \omega)}
$$

where $Y_{1}(\mathrm{j} \omega)$ represents the DFT of the output signal $y_{1}(k)$. The estimated frequency responses are compared with an approximated model for the inner con- 
a) DX

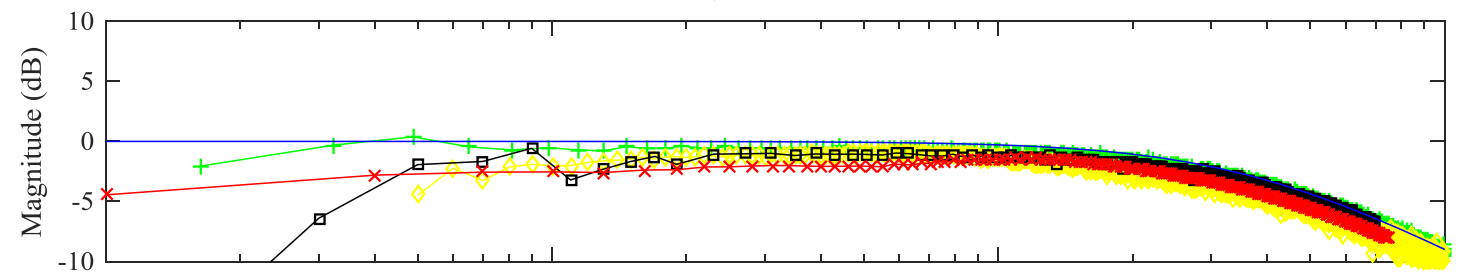

b) DY

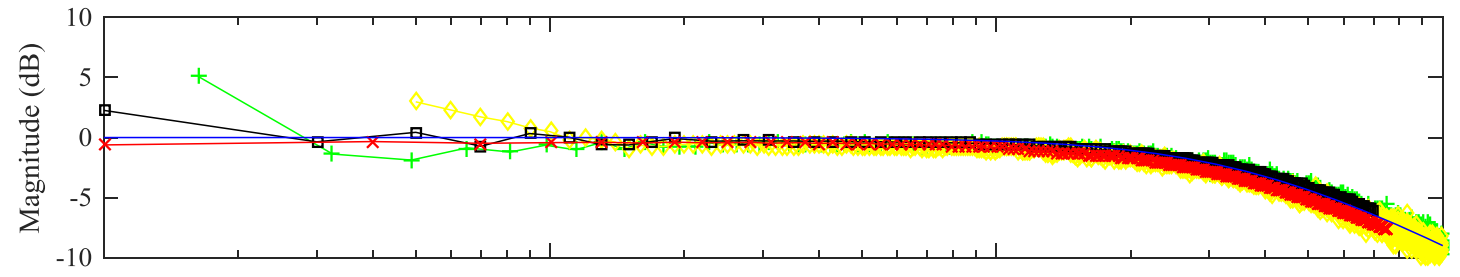

c) NX

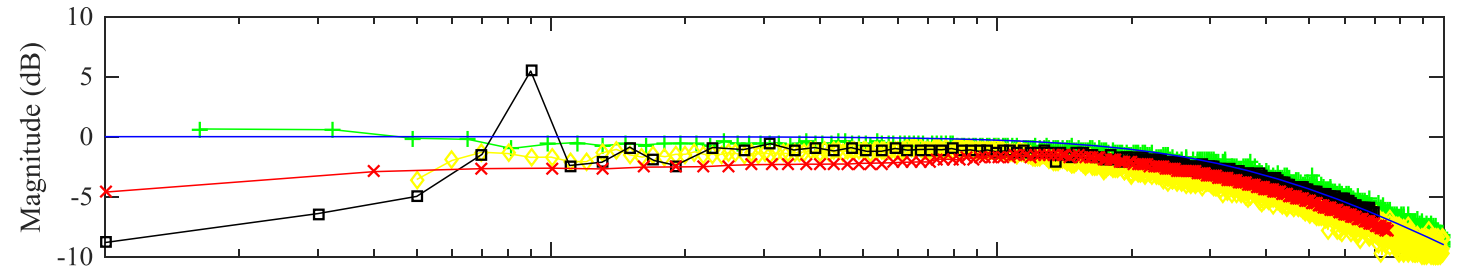

d) NY

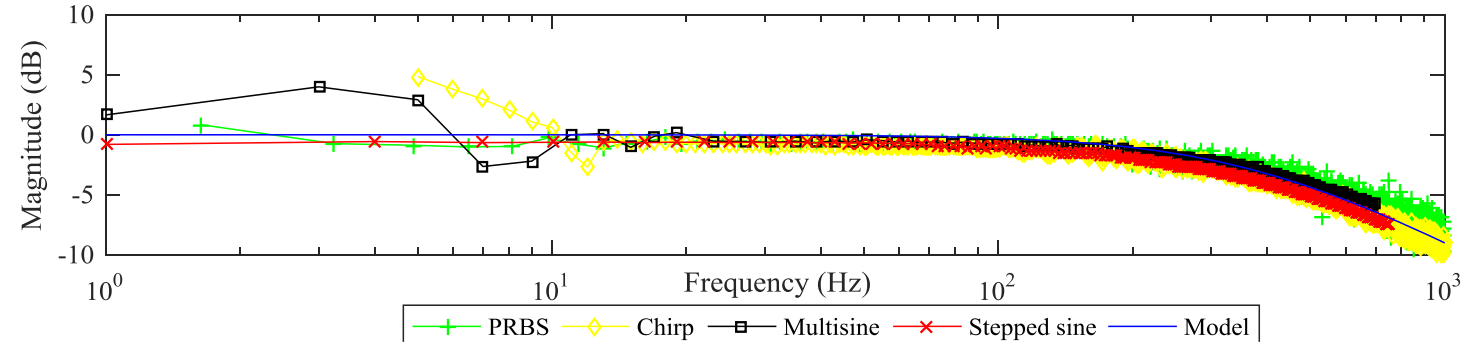

Figure 5: Comparison of the inner current control loop transfer functions. DX and DY are the drive-end x-axis and $\mathrm{y}$-axis, respectively. NX and NY are the non-drive-end $\mathrm{x}$-axis and $\mathrm{y}$-axis, respectively.

trol loop expressed as

$$
G_{m}(\mathrm{j} \omega)=\frac{\omega_{1}}{\mathrm{j} \omega+\omega_{1}}
$$

where $\omega_{1}$ is the cutoff frequency, in this case $380 \mathrm{~Hz}$. This first-order approximation can be considered accurate enough for the inner current control loop model. A comparison between the frequency response functions obtained with the PRBS-, chirp-signal-, multisine-, and stepped-sine-based identification experiments and an approximated model based on (11) of the inner current control loop are shown in Fig. 5.

It can be seen that all the identification results are in good correspondence with the approximated model. On average, above the $100 \mathrm{~Hz}$ frequency, all excitation signals yield similar results. As expected, the largest discrepancy between the estimated frequency responses is observable in the frequency range below $100 \mathrm{~Hz}$.
Especially, on the non-drive-end and drive-end x-axes (NX and DX), the multisine and stepped sine produce different results in this frequency range compared with the results obtained from other experiments. This can be explained by the difference between the excitation signals, in this case the amplitudes in this specific frequency region. Nevertheless, the results obtained from different identification experiments are in a satisfactory agreement, thereby indicating that similar system dynamics can be estimated.

\subsection{Position and Current Stiffness Identification}

In this paper, the identification results are validated by estimating a parametric modal model for the rigid part. In general, the parameter estimation of rigid and flexible modal models of AMB-supported rotor systems 
can be treated separately Hynynen (2011) and Gahler et al. (1997). The general analytical parametric model for the rigid modes can be expressed in the form Hynynen (2011)

$$
G_{\mathrm{r}}(\mathrm{j} \omega)=\frac{\left(s^{2}-z_{1}^{2}\right)}{\left(s^{2}-p_{1}^{2}\right)\left(s^{2}-p_{2}^{2}\right)},
$$

where $z$ and $p$ represent the zeros and poles of the system. The rigid model dynamics can be expressed with the position stiffness values $k_{x D}$ and $k_{x N}$ and the current stiffness values $k_{i D}$ and $k_{i N}$, and thus, these are chosen as parameters to be estimated. Moreover, by considering certain fixed system dynamics, (12) can be derived for the drive-end $x$ - and $y$ - axis as

$$
G_{\mathrm{r}, \mathrm{D}}(\mathrm{j} \omega)=\frac{k_{i D} \cdot d\left(s^{2}-c\left(k_{x D}+k_{x N}\right)\right)}{\left(s^{2}-a\left(k_{x D}+k_{x N}\right)\right)\left(s^{2}-b\left(k_{x D}+k_{x N}\right)\right)},
$$

and correspondingly, for the non-driven end $x$ - and $y$ axes

$$
G_{\mathrm{r}, \mathrm{N}}(\mathrm{j} \omega)=\frac{k_{i N} \cdot d\left(s^{2}-c\left(k_{x D}+k_{x N}\right)\right)}{\left(s^{2}-a\left(k_{x D}+k_{x N}\right)\right)\left(s^{2}-b\left(k_{x D}+k_{x N}\right)\right)} .
$$

It is pointed out that now the parameters $a, b, c$, and $d$ describe the above-mentioned fixed system dynamics, that is, the parameters contain information about the sensor and actuator locations and transformation of the modal parameters into physical coordinates. In this paper, the fixed denominator parameters for the AMB system under study are $a=0.05453$ and $b=0.08494$ for both the drive-end and non-drive-end rigid models. The numerator fixed parameters $c$ and $d$, instead, are slightly different for the drive end $(c=0.06880, d=$ $0.1162)$ and non-drive end $(c=0.06873, d=0.1168)$, respectively. All these fixed parameters are constant assuming that changes are only made to the position and current stiffness values and are thus different for different rotor-bearing models.

By selecting $M$ frequency points, the best fit for the position and current stiffness values of the analytical models can be iteratively searched by minimizing the error function. Naturally, the general form of the error function is the same for all axes, and thus, here the drive-end $x$-axis is given as an example

$$
J_{\mathrm{DX}}(\boldsymbol{\theta})=\sum_{i=1}^{M} w_{i}\left|G_{\mathrm{e}, \mathrm{DX}}\left(\mathrm{j} \omega_{i}\right)-G_{\mathrm{r}, \mathrm{D}}\left(\mathrm{j} \omega_{i}, \boldsymbol{\theta}\right)\right|^{2},
$$

where $\boldsymbol{\theta}=\left[\begin{array}{llll}k_{i D} & k_{x D} & k_{i N} & k_{x N}\end{array}\right]$ is the parameter vector to be estimated, $G_{\mathrm{e}, \mathrm{DX}}(\mathrm{j} \omega)$ are the experimental frequency response data, and $w_{i}$ is a weighting function. Motivated by the method presented in Wroblewski et al. (2012), where flexible modes of a FEM model have been updated based on identification experiments, in this paper, the rigid model is fitted by considering the well-known Nelder-Mead function minimization method and excitation frequencies in the frequency range of only $\{1,200\} \mathrm{Hz}$ as these frequencies correspond mainly to the rigid model. Both the driveend $x$ - and $y$-axes and the non-drive-end $x$ - and $y$-axes are fitted the same, and thus, the total error function is written as

$$
J_{\mathrm{tot}}(\boldsymbol{\theta})=J_{\mathrm{DX}}(\boldsymbol{\theta})+J_{\mathrm{DY}}(\boldsymbol{\theta})+J_{\mathrm{NX}}(\boldsymbol{\theta})+J_{\mathrm{NY}}(\boldsymbol{\theta}) .
$$

For the PRBS, $M=122$ frequency points are used, for the chirp $M=196$, for the multisine $M=54$, and for the stepped sine $M=67$. The weighting function $w_{i}$ is selected so that the frequencies in the range of $\{10$, $100\} \mathrm{Hz}$ are weighted with 2.5 and the other frequencies with 1.

The identified position and current stiffness values are also compared with static measurements with a force gauge. A force gauge manufactured by PCE Instruments, type PCE-FB2k, model H3-C3-300kg-3B, is used. This force gauge has a measuring range up to $2000 \mathrm{~N}$ and a resolution of $0.5 \mathrm{~N}$. For the measurement, the rotor is connected with an elastic rope to a crane. The force gauge is connected between the rope and the crane. A comparison of the identified position and current stiffness values between all the excitation signals and the static measurements is given in Table 1.

Table 1 shows that the results obtained by using different excitation signals provide very similar results for the current stiffness at both the drive and non-drive ends. Compared with the initial assumption in the FEM modeling there is an about $55 \%$ increase. The identification methods provide similar results for the position stiffness at the drive end, but the value of the static measurement is around 2.5 times higher than the identified results. At the non-drive end, the position stiffness value has a higher variation between the identification methods, but the variation is within a reasonable range. Again, the value of the static measurement is around 2.5 times as high as with the identification methods. This can be attributed to the nonlinear behavior of the position stiffness. The applied differential driving mode effectively linearizes the current stiffness but not the position stiffness. An analytical example of this is shown in Fig. 6, where Fig. 6 a) shows the behavior of the current force relation (current stiffness) and Fig. $6 \mathrm{~b}$ ) the behavior of the position force relation (position stiffness).

To further analyze the estimated parameters, a few remarks should be made. First, the position stiffness 
a)
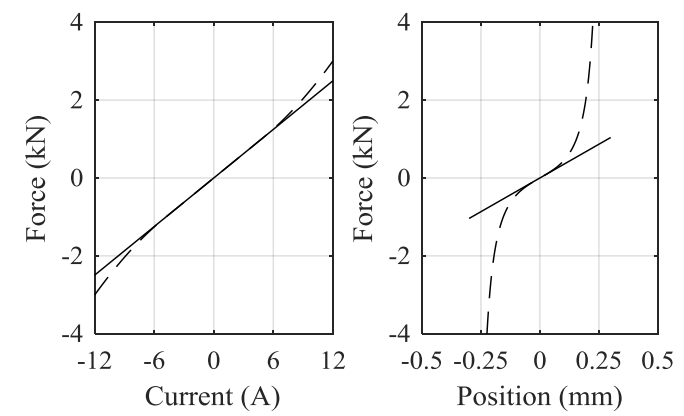

Figure 6: Analytical example of the linearization of (a) current force (current stiffness) and (b) position force (position stiffness) relation. The dotted line indicates the modeled nonlinear behavior and the solid line the assumed linear behavior.

is influenced by the steady state in the inner loops, which can obscure the error in the estimation. Still, it should be noticed that the estimated values are closer to the initial FEM model assumption than the ones obtained from the static position stiffness measurement. Second, the excitation signal has been superposed to the current control reference signal, which has an influence on the position stiffness estimation. For the current stiffness identification, instead, the frequencydomain identification seems to be appropriate as the results with the tested excitation signals are close to the static measurement. The challenges of identifying the stiffness (presented in Table 1), the combined inner current control loop (including PWM), and mechanical runout Kim and Lee (1997) contribute to the dc gain errors of the frequency-dependent plots Fig. 4. Nevertheless, the estimated parameters from the frequency domain observations are reasonable, and a good correspondence between the identification experiments is obtained, indicating that all of the studied excitation signals are suitable for identification of such system dynamics.

\section{Conclusion}

All the excitation signals presented in this paper, viz. the PRBS, chirp signal, multisine, and stepped sine, were found to be suitable for the identification of the rotor-bearing system, inner current control loop, and the position and current stiffness of an AMB system. The results indicated that all of the compared excitation signals are applicable for AMB system identifi-
Table 1: Estimated Position and Current Stiffness Compared with the Initial FEM Model and Static Measurement with A Force Gauge

\begin{tabular}{lllll}
\hline \hline Method & $k_{x D}[\mathrm{kN} / \mathrm{m}]$ & $k_{i D}[\mathrm{~N} / \mathrm{A}]$ & $k_{x N}[\mathrm{kN} / \mathrm{m}]$ & $k_{i N}[\mathrm{~N} / \mathrm{A}]$ \\
\hline Initial FEM Model & 97.30 & 19.4 & 97.30 & 19.4 \\
PRBS & 148.00 & 31.04 & 132.00 & 31.04 \\
Chirp & 156.00 & 31.04 & 126.00 & 31.04 \\
Multisine & 156.00 & 31.04 & 148.00 & 31.04 \\
Stepped sine & 156.00 & 31.04 & 155.00 & 31.03 \\
Static measurement & 390.21 & 30.00 & 390.21 & 30.00 \\
\hline \hline
\end{tabular}

cation, and more importantly, based on the identified frequency responses and estimated stiffness parameters they provide similar results.

To sum up, no final decision could be made on the 'best' excitation signal for AMB system identification. The decision on the signal to be applied could be made based on the considerations presented in this paper. The authors propose the PRBS for first and fast identification of the system in the commissioning phase, and the stepped sine to obtain more accurate results especially at higher and specific frequencies. The analysis of the excitation signals and their selection have a significant impact on identification, diagnostics, and monitoring of various levitation systems. The obtained results are generalizable to a wide variety of such control systems.

Future research could focus on testing the indirect and joint input-output approach for transfer function identification. Further, the system could be modified so that the excitation could be applied at the position control input. When this modification has been made, the position stiffness identification in the frequency domain could be repeated.

\section{References}

Aenis, M., Knopf, E., and Nordmann, R. Active magnetic bearings for the identification and fault diagnosis in turbomachinery. Mechatronics, 2002. 12(8):1011-1021. doi:10.1016/S0957-4158(02)000090 .

Ahn, H.-J., Lee, S.-W., Lee, S.-H., and Han, D.-C. Frequency domain control-relevant identification of mimo amb rigid rotor. Automatica, 2003. 39(2):299 - 307. doi:10.1016/S0005-1098(02)00203-0.

Fang, J., Zheng, S., and Han, B. Amb vibration control for structural resonance of double-gimbal control moment gyro with high-speed magnetically suspended rotor. IEEE/ASME Trans. Mech., 2013. 18(1):32-43. doi:10.1109/TMECH.2011.2161877. 
Gahler, C., Mohler, M., and Herzog, R. Multivariable identification of active magnetic bearing systems. JSME International Journal Series C, 1997. 40(4):584-592. doi:10.1299/jsmec.40.584.

Garcia, J., Gomes, A., and Stephan, R. Performance assessment of a self-bearing motor: an application of iso 14839. In Proc. of 14 th Int. Symp. on Mag. Bear. (ISMB). pages 155-158, 2016.

Hynynen, K. Broadband excitation in the system identification of active magnetic bearing rotor systems. Ph.D. thesis, Lappeenranta University of Technology, Lappeenranta, Finland, 2011.

Hynynen, K. and Jastrzebski, R. P. Optimized excitation signals in amb rotor system identification. In IASTED Int. Conf. of Ident., Contr. and Appl. pages 1-6, 2009.

Hynynen, K., Jastrzebski, R. P., and Smirnov, A. Experimental analysis of frequency response function estimation methods for active magnetic bearing rotor system. In Proc. of 12th Int. Symp. on Mag. Bear. (ISMB). pages 40-46, 2010.

Inman, D. J., Kasarda, M., Quinn, D., Bash, T., Mani, G., Kirk, R., and Sawicki, J. T. Magnetic bearings for non-destructive health monitoring of rotating machinery supported in conventional bearings. In Dam. Assess. of Struct. VI, volume 293. pages 383-390, 2005. doi:10.4028/www.scientific.net/KEM.293-294.383.

Jastrzebski, R., Sillanpaa, T., Jaatinen, P., Smirnov, A., Vuojolainen, J., Lindh, T., Laiho, A., and Pyrhonen, O. Automated design of amb rotor systems with standard drive, control software and hardware technologies. In Proc. of 15th Int. Symp. on Mag. Bear. (ISMB). pages 78-85, 2016a.

Jastrzebski, R. P., Vuojolainen, J., Jaatinen, P., Sillanpää, T., and Pyrhönen, O. Commissioning of modular $10 \mathrm{kw}$ magnetically levitated test rig. In 19th Int. Conf. on Elecl. Mach. and Syst. (ICEMS). pages 1-6, 2016b.

Kim, C.-S. and Lee, C.-W. In situ runout identification in active magnetic bearing system by extended influence coefficient method. IEEE/ASME Trans. Mech., 1997. 2(1):51-57. doi:10.1109/3516.558858.

Kulesza, Z. Dynamic behavior of cracked rotor subjected to multisine excitation. Journal of Sound and Vibration, 2014. 333(5):1369 - 1378. doi:10.1016/j.jsv.2013.10.031.
Lanzon, A. and Tsiotras, P. A combined application of $\mathrm{h}$ infin; loop shaping and mu;-synthesis to control high-speed flywheels. IEEE Trans. Control Syst. Technol., 2005. 13(5):766-777. doi:10.1109/TCST.2005.847344.

Larsonneur, R. Principle of Active Magnetic Suspension, pages 27-68. Springer Berlin Heidelberg, Berlin, Heidelberg, 2009. doi:10.1007/978-3-642$00497-1_{2}$.

Noshadi, A., Shi, J., Lee, W. S., Shi, P., and Kalam, A. System identification and robust control of multiinput multi-output active magnetic bearing systems. IEEE Trans. Contr. Syst. Tech., 2016. 24(4):12271239. doi:10.1109/TCST.2015.2480009.

Pintelon, R. and Schoukens, J. Design of Excitation Signals, pages 151-175. Wiley-IEEE Press, 2012. doi:10.1002/9781118287422.ch5.

Quinn, D. D., Mani, G., Kasarda, M. E. F., Bash, T., Inman, D. J., and Kirk, R. G. Damage detection of a rotating cracked shaft using an active magnetic bearing as a force actuator - analysis and experimental verification. IEEE/ASME Trans. Mech., 2005. 10(6):640-647. doi:10.1109/TMECH.2005.859833.

Schuhmann, T., Hofmann, W., and Werner, R. Improving operational performance of active magnetic bearings using kalman filter and state feedback control. IEEE Trans. Ind. Electron., 2012. 59(2):821829. doi:10.1109/TIE.2011.2161056.

Smirnov, A. AMB system for high-speed motors using automatic commissioning. Ph.D. thesis, Lappeenranta University of Technology, Lappeenranta, Finland, 2012.

Tang, E., Han, B., and Zhang, Y. Optimum compensator design for the flexible rotor in magnetically suspended motor to pass the first bending critical speed. IEEE Trans. Ind. Electron., 2016. 63(1):343354. doi:10.1109/TIE.2015.2472534.

Tiwiri, R. and Chougale, A. Identification of bearing dynamic parameters and unbalance states in a flexible rotor system fully levitated on active magnetic bearings. Mechatronics, 2014. 24(3):1011-1021. doi:10.1016/j.mechatronics.2014.02.010.

Vuojolainen, J., Jastrzebski, R., and Pyrhonen, O. Using a pseudorandom binary sequence for rotorbearing system identification in active magnetic bearing rotor systems. In Proc. of 15th Int. Symp. on Mag. Bear. (ISMB). pages 618-625, 2016. 
Wroblewski, A., Sawicki, J., and Pesch, A. Rotor model updating and validation for an active magnetic bearing based high-speed machining spindle. ASME.
J. Eng. Gas Turbines Power, 2012. 134(12):1-6. doi:10.1115/1.4007337. 